\title{
Critical View on the Creep Modelling Procedures
}

\author{
L. KLOC* \\ Institute of Physics of Materials AS CR, v.v.i, Žižkova 22, 61662 Brno, Czech Republic
}

\begin{abstract}
Process of creep deformation is rather complex, consisting of many interconnected subprocesses, mainly: (i) the creep strain itself, based on dislocation mobility as well as grain boundary activity, (ii) development of dislocation substructure (work strengthening and dynamic recovery), (iii) development of phase structure (phase transformations, precipitation, particle coarsening, etc.), and (iv) nucleation and development of voids and microcracks, i.e. creep damage. The creep experiments are time consuming and expensive, moreover, it is not possible to make experiments under the service conditions of particular materials due to very slow creep strain, the process seems to be ideal field for computer modelling. The experimental data are obviously available for the steady conditions only, so the effects of varying conditions during startup or shutdown of the components can be described by modelling. The model of creep deformation is obviously based on the so-called "creep constitutive equation", which should describe the strain rate dependence on stress, temperature and some other variables. Nevertheless, the comprehensive physical description of all the above mentioned processes is still missing. This paper should illustrate the shortcomings of most "creep constitutive equations", confronting them to some experimental results on common structural materials under non-steady loading conditions.
\end{abstract}

DOI: 10.12693 /APhysPolA.128.540

PACS: $62.20 . \mathrm{Hg}, 87.10 . \mathrm{Kn}, 81.40 . \mathrm{Lm}$

\section{Introduction}

Demands for further improvement of the efficiency of the thermal power plants could be satisfied by higher steam temperatures, but potential of ferritic steels is exhausted. Austenitic steels with better creep strength and corrosion resistance are then planned to replace them, but they are susceptible to thermal fatigue due to higher thermal expansion and lower thermal conductivity. Thermal fatigue is critically dependent on the temperature and stress fields in massive parts during changes of operational conditions. Finite element method (FEM) modelling seems to be very powerful tool to solve such problem.

TABLE

Comparison of the FEM method demands and available creep data.

\begin{tabular}{c|c}
\hline \hline FEM demands & Available data \\
\hline $\begin{array}{c}\text { variable stress } \\
\text { variable temperatures } \\
\text { wide range of stresses } \\
\text { including low stress regime }\end{array}$ & constant stress \\
constant temperatures
\end{tabular}

This is only one example explaining the growing interest in FEM modelling of processes including an important role of creep. Precise mathematical description of material behaviour is necessary to obtain realistic results of modelling. Unfortunately, this is not the case of creep processes. Demands of creep process description by modelling software is compared to typical properties data available from experiments in the Table. It is clear that

\footnotetext{
*e-mail: kloc@ipm.cz
}

conventional creep experiments cannot provide information needed for modelling, where external conditions are variable. Since the conventional creep experiments are standardized, it is generally believed that they can provide complete information on the creep behaviour of the material. But the "constitutive equations", derived from the conventional creep tests results are not able to describe creep behaviour under variable loading conditions correctly.

The constitutive creep equations taken from the textbooks [1] use to be implemented in FEM software systems. All the equations were published very long time ago and are fully empirical, trying to describe existing experimental creep curves precisely. Nevertheless, there are several reasons why these equations cannot be used for realistic modelling of creep phenomena.

Most frequently the equations have differential form

$$
\dot{\epsilon}=f\left(\sigma, T, c_{i}\right) \text {, }
$$

where $\dot{\epsilon}$ is strain rate, $T$ is temperature, $\sigma$ is applied stress and $c_{i}$ are material constants, which can be derived by fitting the equation on the experimental data. Integral form

$$
\epsilon=f\left(\sigma, T, t, c_{i}\right)
$$

is also sometime used, where $\epsilon$ is creep strain and $t$ is time. In better case, there is some attempt to reflect the fact of strain rate changes during the creep process, adding a time $t$ or strain $\epsilon$ to the differential form of Eq. (1), giving

$$
\dot{\epsilon}=f\left(\sigma, T, t, c_{i}\right) \quad \text { or } \quad \dot{\epsilon}=f\left(\sigma, T, \epsilon, c_{i}\right) .
$$

But from the physical point of view, the creep process is described as

$$
\dot{\epsilon}=f\left(\sigma, T, s_{i}\right),
$$

where $s_{i}$ are parameters describing the general microstructural state of the material, which is not constant, but depends on the whole loading history and evolves 
continuously. Important part of the microstructural state is a field of internal stresses, which can cause negative flow against the direction of the applied stress. Thus, the time and strain are not monotonically dependent variables, as with constant conditions tests, but in the case of variable external conditions they must be treated as independent variables. Unfortunately, most of the above mentioned equations do not possess that capability.

\section{Discussion}

\subsection{Formal errors}

In this subsection, various types of frequently occurring errors or problems are briefly described.

- Non-zero strain rate for zero stress. In some cases, the equation yields positive strain rate even for zero or negative applied stress. This holds mainly for equations, where the stress dependence of the creep rate is described by exponential term $\dot{\epsilon} \propto \exp \left(c_{i} \sigma\right)$. This may provide acceptable description of conventional creep test at rather high stresses, but it can produce completely wrong result with modelling, where the stress in some elements can easily drop to zero.

- Missing temperature dependence. Constant temperature only is not very interesting for the modelling.

- Secondary stage only. In most structural material, the secondary stage is reduced to relatively narrow region of the minimum creep rate, while the creep curves are dominated by primary and/or tertiary stages. It is not worthwhile to limit modelling process to the very specific state of the material.

- Primary stage only. This restriction is not as limiting as the previous, but can be used practically for modelling of brand new material. It is difficult to avoid situation, where the ever decrease of creep rate starts to become unrealistic.

- Too many parameters. With these equations, problems with parameter correlations can be expected during fitting process. Very extensive data pool is needed to fit these equations with reasonable errors of all parameters.

\subsection{Temperature dependence}

In most equations, the temperature dependence is described by the Arrhenius type term, that is $\dot{\epsilon} \propto$ $\exp (-Q /(R T))$ assuming the apparent activation energy $Q$ to be constant. But in fact, with many interesting structural materials, the value of apparent activation energy $Q$ is stress dependent due to changes in deformation mechanism [2]. This is illustrated in Fig. 1. During modelling, the stress can easily enter the range where the temperature dependence parameter is invalid.

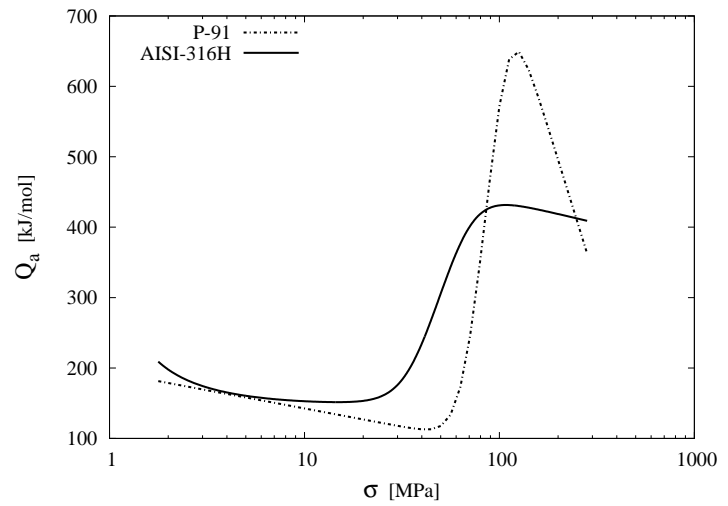

Fig. 1. Stress dependence of apparent activation energy of P-91 and AISI-316H steels. Figure reproduced from [2].

\subsection{Stress dependence}

Since the equations were derived from constant stress or constant load test data, they do not reflect the transient stages after stress changes due to changes in internal stress field. Internal stress field play important role mainly in lower stress range, which is important for industrial applications of materials [3]. Part of creep curve, illustrating these transients is in Fig. 2. Data are taken

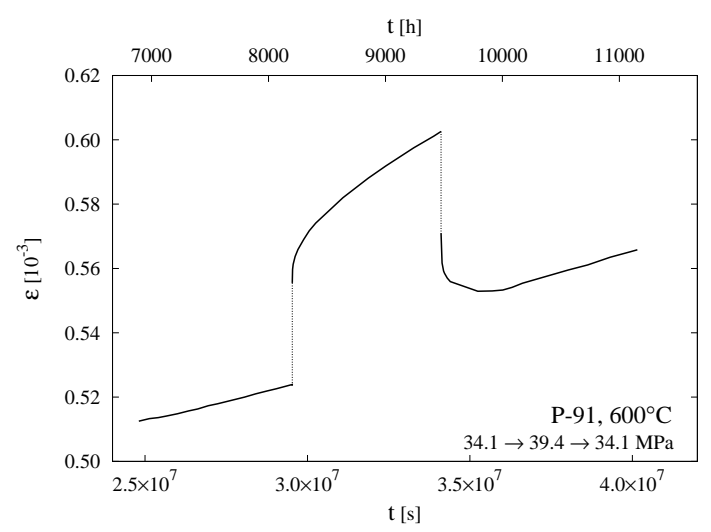

Fig. 2. Part of creep curve of p-91 steel at $600{ }^{\circ} \mathrm{C}$ with stress change from $34.1 \mathrm{MPa}$ to $39.4 \mathrm{MPa}$ and back.

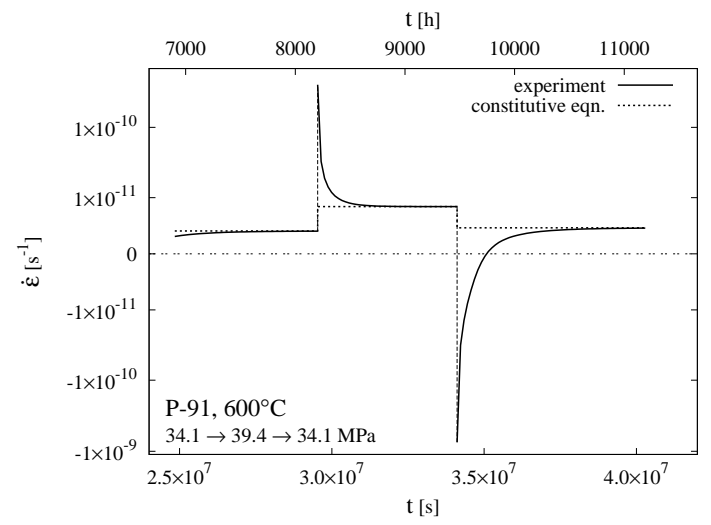

Fig. 3. Part of creep curve from Fig. 2 in creep rate vs. time coordinates. 


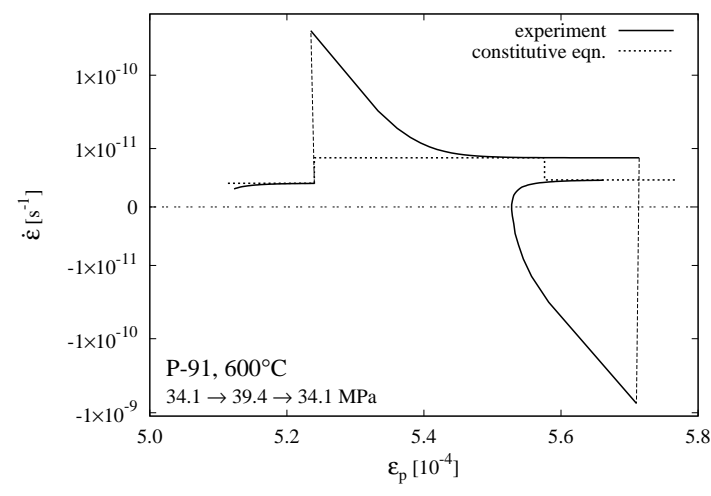

Fig. 4. Part of creep curve from Fig. 2 in creep rate vs. creep (non-elastic) strain coordinates.

from [4], where more details can be found. In Figs. 3 and 4 , the same creep curve is depicted as creep rate vs. time or creep rate vs. strain, respectively. In the figures, the behaviour of all the analyzed equations is also shown. It is clear that in the case of stress change the equations simply "switch" from one creep curve to other and back, completely ignoring the transient stage. Thus, after stress reduction, the equations assume some positive creep flow, while negative flow with much higher magnitude occurs in reality.

As a starting point to solve this problem, model developed in [5] can be used. The equation describing the creep rate dependence on stress is

$$
\dot{\epsilon}=\frac{c_{1} c_{3}}{c_{1}+c_{2}} \sinh \left[c_{5}\left(\sigma-\frac{\epsilon-c_{4} \sigma t}{c_{1}}\right)\right]+c_{4} \sigma,
$$

where, unlike the obvious creep equations, $\epsilon$ is total strain including elastic part. Though this is largely simplified assuming the transient effects are caused exclusively by internal stresses, it provides much more realistic description of the real behaviour of the material than any of the above mentioned "constitutive" equations.

\section{Conclusions}

The constitutive equations for creep built in the FEM systems in most cases cannot be used for realistic predictive description of the creep behaviour of materials under varying stress and temperature conditions. The equations were derived to describe conventional creep experiments under constant conditions, thus ignoring the transient effects due to internal stresses. It has been demonstrated that the transient effects and deformation mechanism changes cannot be neglected, mainly under conditions interesting for modelling. Further, there is a number of additive less serious problems with the equations, too.

\section{Acknowledgments}

This work was supported by the Ministry of Industry and Trade of the Czech Republic under the Project no. FR-TI4/406.

\section{References}

[1] R.K. Penny, D.L. Marriott, Design for Creep, McGraw-Hill, London 1971, p. 192.

[2] L. Kloc, V. Sklenička, J. Ventruba, Mater. Sci. Eng. A 319-321, 774 (2001).

[3] K. Milička, F. Dobeš, Eng. Mech. 5, 165 (1998).

[4] L. Kloc, V. Sklenička, Mater. Sci. Eng. A 387-389, 633 (2004).

[5] L. Kloc, J. Phys. Conf. Series 240, 012086 (2010). 